\title{
Gait disturbances in football, rugby players and skiers following anterior cruciate ligament reconstruction
}

\author{
M.C. Zielinski ${ }^{1}$, R. Twycross Lewis ${ }^{1}$, R. Woledge ${ }^{1}$, N. Maffulli ${ }^{1}, 2,3$ \\ 1 Queen Mary University of London, Barts and the London School of Medicine and Dentistry, Centre for Sports \\ and Exercise Medicine, Biomechanics Laboratory, Mile End Hospital, UK \\ 2 Department of Musculoskeletal Disorder, Faculty of Medicine and Surgery, University of Salerno, Salerno, Italy \\ 3 Institute of Science and Technology in Medicine, Keele University School of Medicine, Stoke on Trent, UK
}

\section{CORRESPONDING AUTHOR:}

Nicola Maffulli

Department of Musculoskeletal

\section{Disorders}

Faculty of Medicine, Surgery

and Dentistry

University of Salerno

Via Allende

Baronissi (Salerno)

ITALY

Email: n.maffulli@qmul.ac.uk

DOI:

10.32098/mltj.01.2019.19

\begin{abstract}
SUMMARY
Background. Patients who have undergone anterior cruciate ligament (ACL) reconstruction surgery may exhibit post-operative lower limb gait disturbances. Design. Laboratory in vivo controlled study. Methods. Kinematics and kinetics parameters of knee motion were measured in post-ACL reconstruction participants $(\mathrm{n}=5 ; \mathrm{m}=4 / \mathrm{f}=1$, age 30.6 years, height $179.2 \mathrm{~cm}$, weight $80.4 \mathrm{~kg})$ and control participants $(\mathrm{n}=10 ; \mathrm{m}=6 / \mathrm{f}=4$, age 30.3 years, height $173.5 \mathrm{~cm}$, weight $65.3 \mathrm{~kg}$ ). Kinematics and kinetics were recorded for normal gait, 'cutting' gait and 'weaving' gait ( $\dot{x}$ $=3$ per gait type) using instrumented motion analysis and ground embedded force plates. Between groups, differences in knee joint alignment were determined in the frontal plane for normal gait, and in the coronal plane for cutting and weaving. Five of the subjects were recruited for this study had an ACL reconstruction in the past (at least two years before the test), and ten were part of a control group. Each subject performed three different tasks with both legs on a force plate: normal walking, a weaving movement, and a cutting movement. Kinematic and kinetic data were collected with four optoelectronic cameras and two force plates. Comparisons were made in the frontal plane for walking and in the coronal plane for cutting and weaving between the reconstructed knee and the contralateral, healthy knee in the late stance period. Results. There were no significant differences between the ACL reconstructed knees and the contralateral healthy knees in the walking task $(\mathrm{p}=0.27-0.49)$. Knee coronal plane rotation showed between group similarity (cutting $=21.82^{\circ}$ vs $11.29^{\circ}, \mathrm{p}=0.175$; weaving $=17.88$ vs $21.34^{\circ}, \mathrm{p}=0.406$ ). Conclusions. There is an increased rotation of the knee during walking and cutting in ACL reconstructed knees when compared to the contralateral knee, although this difference was not statistically significant.
\end{abstract}

\section{KEY WORDS}

anterior cruciate ligament; biomechanics; football; kinetics; kinematic abnormalities; reconstruction; rugby

\section{BACKGROUND}

Even after intensive rehabilitation following anterior cruciate ligament reconstruction (ACLR), these patients are at a greater risk of reinjury and knee osteoarthritis $(1,2)$. No investigation has provided clear scientific evidence that ACL reconstructed patients do return to a normal gait pattern (3). Movement asymmetries are pervasive following ACL injury and reconstruction, and have been reported for up to 2 years after surgery (4). Athletes with multiplanar biomechanical asymmetries at the hip and knee at the time of return to sport were at least three times more likely to incur a second ACL injury within the next year than those without these asymmetries (5). Individuals who have had ACLR exhibit altered knee kinematics and kinetics during dynamic tasks such as side-step cutting and landing, even though they have been released to full participation in sport or full activity $(6,7)$. Altered movement patterns have been suggested to be an instigating factor in the initiation and development of osteoarthritis in the ACL-injured knee as well as a risk factor for future reinjury $(5,8)$. There still exists 
clinical uncertainty as to whether the ACL reconstructed knee can fully return to its preinjury functional capacity (9). This study analysed the biomechanics of the knee while walking, weaving and cutting in ACL reconstructed and fully rehabilitated participants, to detect kinematics and kinetics abnormalities in their knees. We hypothesised that there is a difference between the ACL reconstructed knee and the contralateral knee (non-operated knee) while walking, and that there is a difference in knee rotation while doing cutting and weaving movements between the injured knees and the non-operated ones.

\section{METHODS}

\section{Subjects}

A total of 15 participants (table I) were recruited. Five participants $(\mathrm{m}=4 / \mathrm{f}=1$, age 30.6 , height 179.2 , weight 80.4, BMI 24.8, Lysholm Score 91.2, time from surgery 6.2 years, years of sports experience 16.2) had undergone an ACL reconstruction; all of them were fully rehabilitated, and back to their chosen sports. All participants met our inclusion criteria: football players, rugby players and skiers had more than the minimum of 2 years of experience in their sport (range between 10 and 20 years), their age ranged between 20 and 45 years, they all had a confirmed ACL reconstruction surgery in the past (confirmed by an MRI and their scar), and were completely rehabilitated. Ten healthy recreational athletes $(\mathrm{m}=6 / \mathrm{f}=4$, age 30.3 , height 173.5, weight 65.3, BMI 21.27) without any knee pathology were recruited as controls.

Participants were excluded from the study if they had less than 2 years of sports experience, had knee contracture, radiographic evidence of patellar osteoarthritis which, in the opinion of the treating physician, would have preclude enrolment, history of patellar instability, tight iliotibial band or iliotibial band syndrome, femoral or tibial bony deformity, bone metabolic disease, diseases affecting the connective tissue or muscle and any cardiovascular disease.

\section{Ethics}

This study meets the ethical standards of the journal (10). This research study was approved by the Queen Mary University of London (QMUL) Ethics Committee. Each subject received a detailed information sheet and a signed consent form before participating.

\section{Procedures}

All participants performed three tests. All procedures were performed in the Human Performance Laboratory of QMUL. All participants answered a questionnaire and completed a Lysholm Scoring questionnaire before performing the tests.

The first-test was a standardized walking protocol (figure 1) to establish a baseline for each participant's normal gait. During this task, the participant was required to walk normally in a straight line over the force plates and stop one meter after crossing them.

The second test was a cross over movement called weaving (figure 2). The participant walked towards the force plate. Once arriving at the force plate, the stance leg was supporting the body as the person rotated internally over the knee. When walking to the left side, the participant used the left leg as the stance leg on the force plate and cross over with the right leg. When walking to the right side, the participant used the right leg as a stance leg and crossed over with the left leg.

Table I - Demographics.

\begin{tabular}{llll}
\hline & ACL reconstructed participants (mean) & Control group (mean) & t-test (p value) \\
\hline age & $30.6(28-33)$ & $30.3(26-38)$ & 0.433 \\
\hline sex & $\mathrm{m}=4, \mathrm{f}=1$ & $\mathrm{~m}=6, \mathrm{f}=4$ & 0.23 \\
\hline height $(\mathbf{c m})$ & $179.2(173-186)$ & $173.5(158-195)$ & 0.157 \\
\hline weight $(\mathbf{k g})$ & $80.4(58-93)$ & $65.3(47-95)$ & 0.040 \\
\hline BMI & 24.82 & 21.27 & 0.021 \\
\hline sport experience during the years & 16.2 & $\mathrm{NA}$ & \\
\hline MRI & Yes & $\mathrm{NA}$ & \\
\hline rehab & 15.6 & $\mathrm{NA}$ & \\
\hline time from surgery & $6.2(3-9)$ & $\mathrm{NA}$ & \\
\hline Lysholm score & $91.2(83-100)$ & 100 & different types of sport \\
\hline sport & football $(2)$, rugby $(2)$, skiing $(1)$ &
\end{tabular}



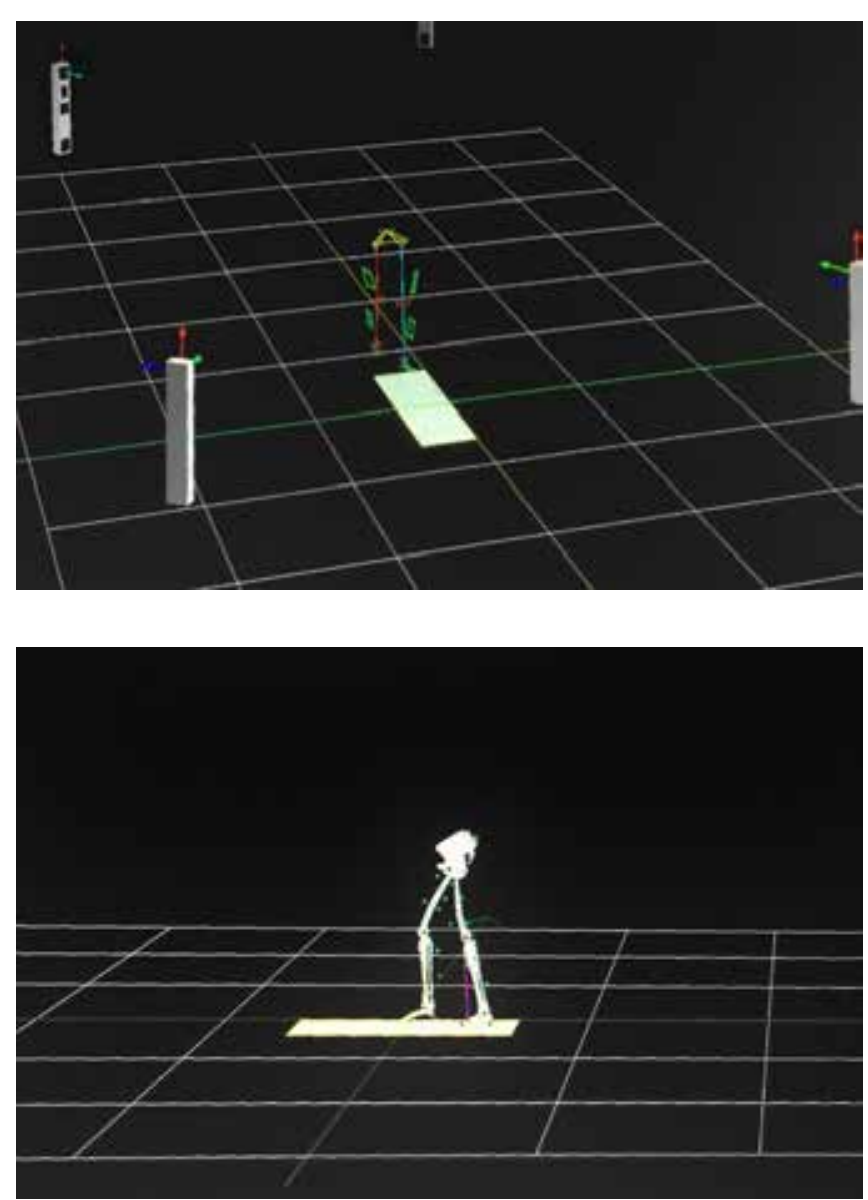

Figure $1 \mathbf{a}$ and $\mathbf{b}$ - Walking task.

The third task was a cutting movement (figure 3). While undertaking the task, the participant had to walk straight towards the force plate. The participant put the contralateral leg as a stance leg on the force plate to support the body while the ipsilateral knee was in swing and changed direction. For the movement to the left, the right leg was used and the subject then put the right leg on the force plate as a stance leg while performing a cutting movement to the left. For movements to the right side, the left leg was used as a stance leg on the force plate while changing direction to the right.

\section{Data collection}

Kinematics data were collected using the Codamotion ODIN system (Charnwood Dynamics Ltd., Leicestershire, UK) at the Human Performance Laboratory, Centre of Sports and Exercise Medicine, Queen Mary University of London.

A cluster protocol based on the Modified Helen Hayes Protocol (figure 4) was used to place the markers on the
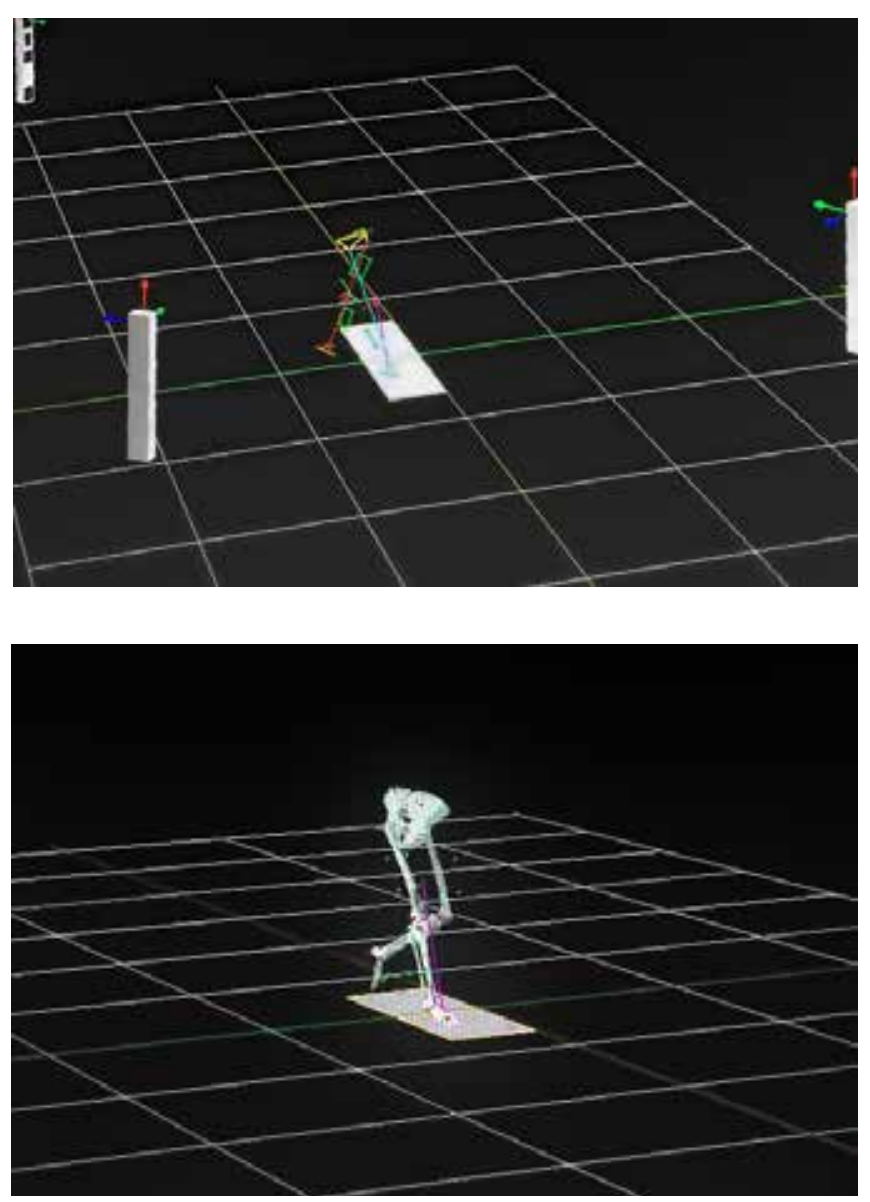

Figure $\mathbf{2} \mathbf{a}$ and $\mathbf{b}$ - Weaving task.

subjects. The markers were placed on the lateral aspect of the 5th metatarsal and the lateral aspect of the calcaneus. Markers were placed on the anterior sacroiliac spine and on the posterior sacroiliac spine. ASIS-ASIS (pelvic width) was measured; ASIS- spine (pelvic depth); knee width and ankle width (medial to lateral epicondyle and malleolus) (figure 5, figure 6, figure 7 )

Movement data from the Codamotion markers were collected at a rate of $200 \mathrm{~Hz}$. Kinetic data were collected from 2 ground embedded force plates (Kistler Type 9281B, Kistler Corporation, Winterthur, Switzerland). Ground reaction force data were collected at a sampling rate of $500 \mathrm{~Hz}$ and graphed in the anterior-posterior axis; medial-lateral axis and superior-inferior axis.

\section{Data analysis}

Data extraction and analysis were made using the Matlab ${ }^{\circledR}$ Software (Matworks ${ }^{\circledR}$, Natrick, Massachusetts, USA) 

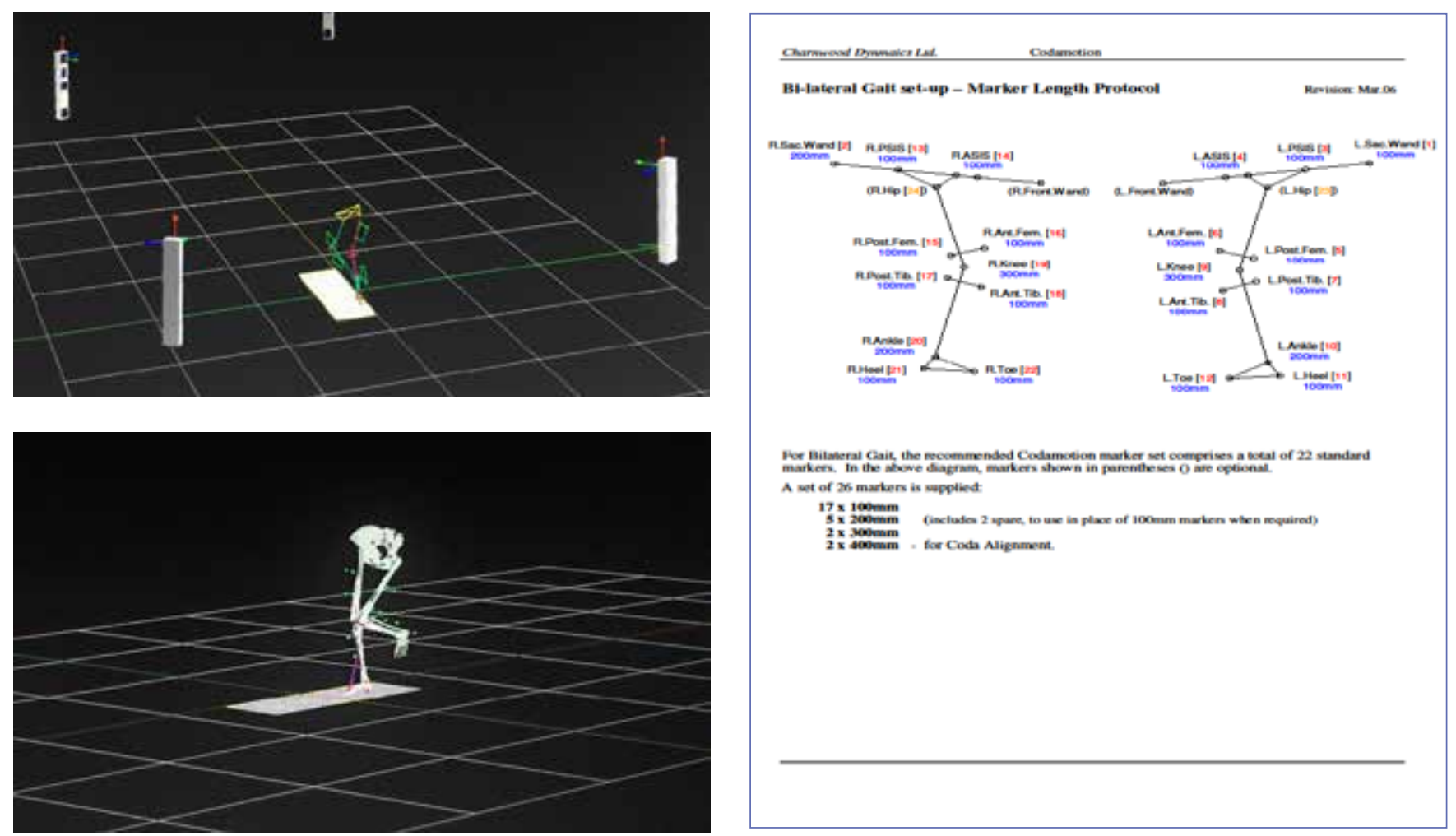

Figure $\mathbf{3} \mathbf{a}$ and $\mathbf{b}$ - Cutting task.

Figure 4 - Helen Hayes Protocol.

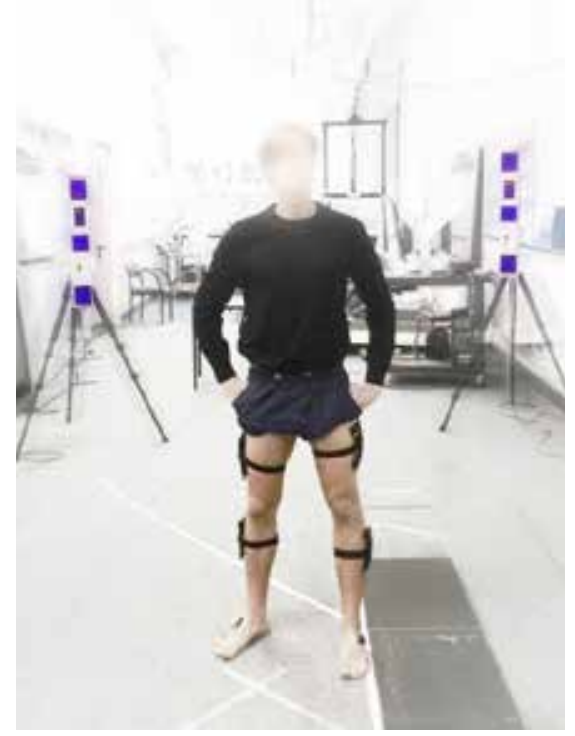

Figure 5 - Data collection: two of the four cameras, two force plates, a participant wearing markers (the left thigh marker placement was corrected for the tests and pelvic markers were also included)

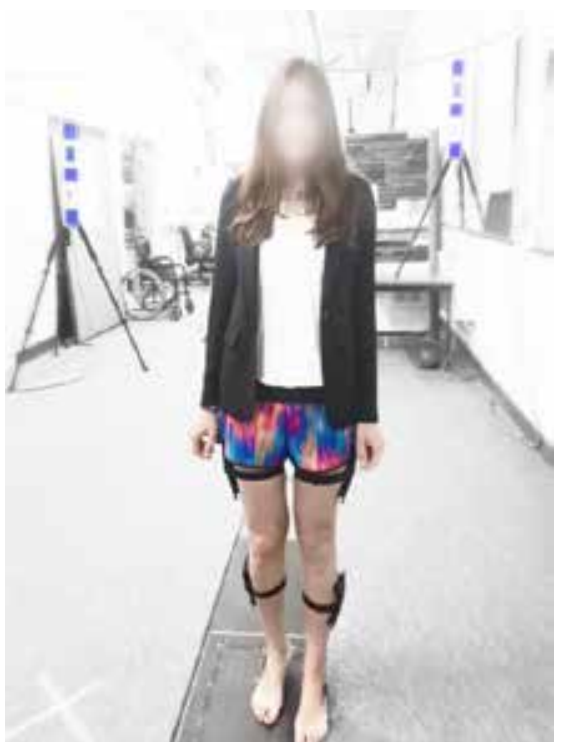

Figure 6 - Data collection: two of the four cameras, two force plates, a participant wearing markers (pelvic markers were also included for the tests).

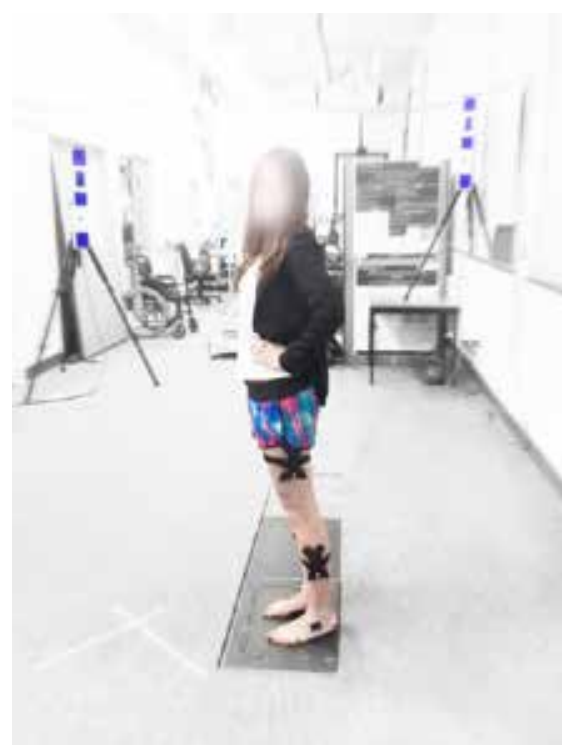

Figure 7 - Data collection: two of the four cameras, two force plates, a participant wearing markers (pelvic markers were also included for the tests). 
version $2013 \mathrm{~b}$. A pre-existing custom written programme was used to measure kinematic data by calculating the joint angles without gait. New custom written programmes were used to calculate joint rotation during cutting and weaving. Kinematics and kinetics abnormalities of the knees were calculated by inverse dynamics from the kinematic data and the ground reaction data measured by the force plates. The ground reaction force vector was divided into its component planes. The distance from the joint centre to the vector throughout the movement was graphed as the change in angle of the knee in the coronal and transverse planes.

Kinematic and kinetic data calculated the transverse plane while walking and the coronal plane during knee rotation when executing cutting and weaving movements in the late stance period (heel off - toe off). A specific gait moment, the late stance phase, was selected for each participant in the Matlab programme for the walking, weaving and cutting task. Given the small sample size, data were unlikely to be normally distributed. Therefore, all data were log transformed to allow parametric analysis. Statistical significance was set at $\mathrm{p}<0.05$.

\section{Statistics}

Statistics were performed using the IBM SPSS software, version 22. Paired t-tests were used to ascertain whether there was a difference in knee rotation during the late stance phase in either cutting or weaving movement between the good limb and the ACL reconstructed limb. Demographic characteristics between groups were compared with the Independent Samples t-test for numerical variables.

For walking, data were analysed using Windows Excel 2013. T-tests were used to obtain the relevant $p$ values during the late stance phase in this task, and ascertain whether there is a significant difference between the limb which had undergone surgery and the healthy limb. The standard error of the mean (SEM) was used to compare the non-operated limb with the ACL reconstructed knee. For normality, dependent variables were tested to compare the shape of the sample distribution to the shape of a normal curve. The Kolmogorov and Shapiro-Wilk tests were used. Reliability was tested using Intraclass Correlation Coefficients (ICC), which were used to assess the consistency or reproducibility of quantitative measurements, taken twice when measuring the same quantity (control groups were measured twice for reliability). For reliability, one single investigator performed the test on two separate occasions to reduce the chances of external confounding variables. Each subject was tested on the same day of the week and at the same hour of the day.

\section{RESULTS}

\section{Lysholm}

The mean of the Lysholm scale for the ACL reconstructed participants was $91.2(83-100)$ with the results ranging from fair to excellent. For the heathy participants, the mean was 100 .

\section{Reliability}

Reliability was tested using ICC tests. Reliability for the left knee rotation in the cutting movement was poor $(\mathrm{p}=0.576$, $\mathrm{ICC}=0.178, \mathrm{df}=6$ ). Reliability for the right was better than for the left knee $(\mathrm{p}=0.176, \mathrm{ICC}=0.551, \mathrm{df}=6$,). Regarding the weaving movement, the reliability was poor for the left leg $(\mathrm{p}=0.363, \mathrm{ICC}=0.258, \mathrm{df}=6)$, but good for the right leg $(\mathrm{p}=0.071, \mathrm{ICC}=0.724, \mathrm{df}=6)$.

\section{Walking}

T-tests were used to analyse the data gained from the walking task. Figure $\mathbf{8}$ shows the results obtained from the frontal plane during the late stance phase and compared the non-operated limbs means to the ACL reconstructed means. There was no significant difference between the two limbs $(\mathrm{p}=0.27-0.49)$.

$\mathrm{P}$ values (green line) found during the walking task (figure 8) compared to standard, significant $\mathrm{p}$ values (red line) sampled during the late stance phase of the gait cycle. Vertical line $=p$ values; horizontal line $=$ gait cycle.

Standard error of the mean deviations (figure 9) were used to compare the non-operated knee with the ACL reconstruc-

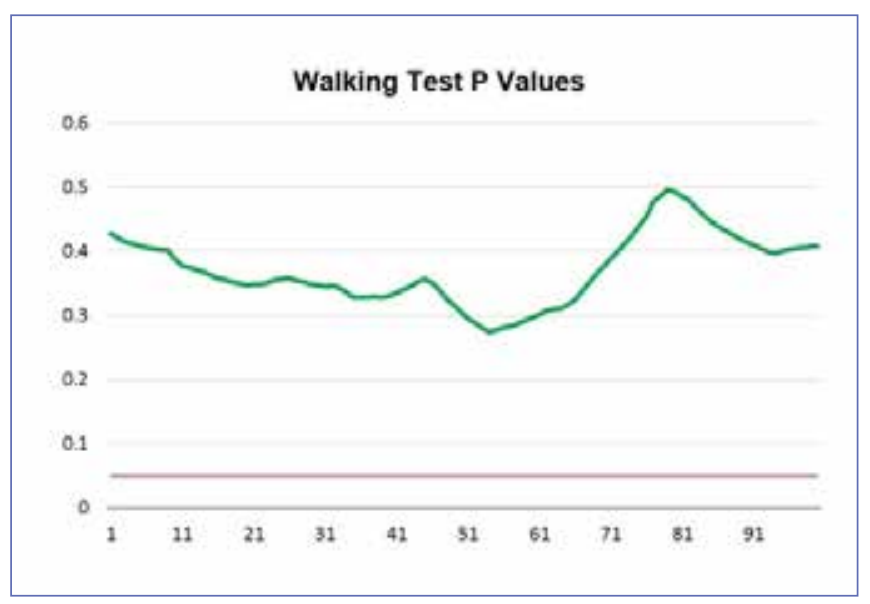

Figure 8 - Walking test $p$ values. 


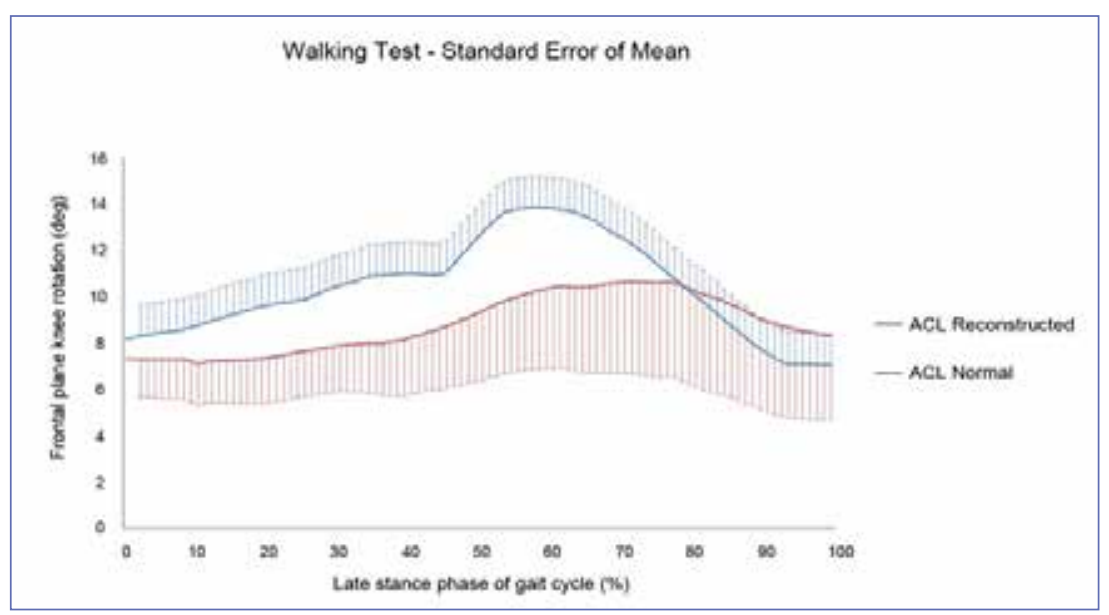

Figure 9 - Walking test, Standard error of Mean (SEM).

ted knee. In the uninjured knee, the rotation of the knee was constant when compared to the injured knee, where there was more variation in the movement.

\section{Cutting}

Paired t-tests were used to analyse the data for ACL participants while cutting. The result was not significant $(t=1.644$, $\mathrm{df}=4, \mathrm{p}=0.175)$. Although the result is not significant, table II shows that ACL reconstructed knees have more rotation $\left(21.82^{\circ}\right)$, normalised to time when compared to the non-operated side $\left(11.29^{\circ}\right.$ ) (figure 10).

\section{Weaving}

When comparing the ACL reconstructed knees with the contralateral knees while undertaking the weaving task (table III), the knee rotation was greater in the non-operated knees $\left(17.88^{\circ}\right.$ and $\left.21.34^{\circ}\right)$. The result was not significant $(\mathrm{t}=0.929, \mathrm{df}=4, \mathrm{p}=0.406)$.

The ACL reconstructed knees and the non-operated knees were normally distributed in both cutting and weaving tasks.

Table II - Knee rotation while performing the cutting test.

\begin{tabular}{lll}
\hline & $\begin{array}{l}\text { ACL reconstructed } \\
\text { knee }\end{array}$ & $\begin{array}{l}\text { Non-operated } \\
\text { knee }\end{array}$ \\
\hline $\begin{array}{l}\text { mean Rotation } \\
\text { (d } \theta / \text { dTime })\end{array}$ & $21.92 \pm 16.07$ & $11.29 \pm 11.86$ \\
\hline $\mathbf{t}$ & 1.644 & \\
\hline $\mathbf{d f}$ & 4 & \\
\hline $\mathbf{p}(\mathbf{2}$ tailed $)$ & 0.175 & \\
\hline
\end{tabular}

Values for rotation are in degrees. No significant differences were found.

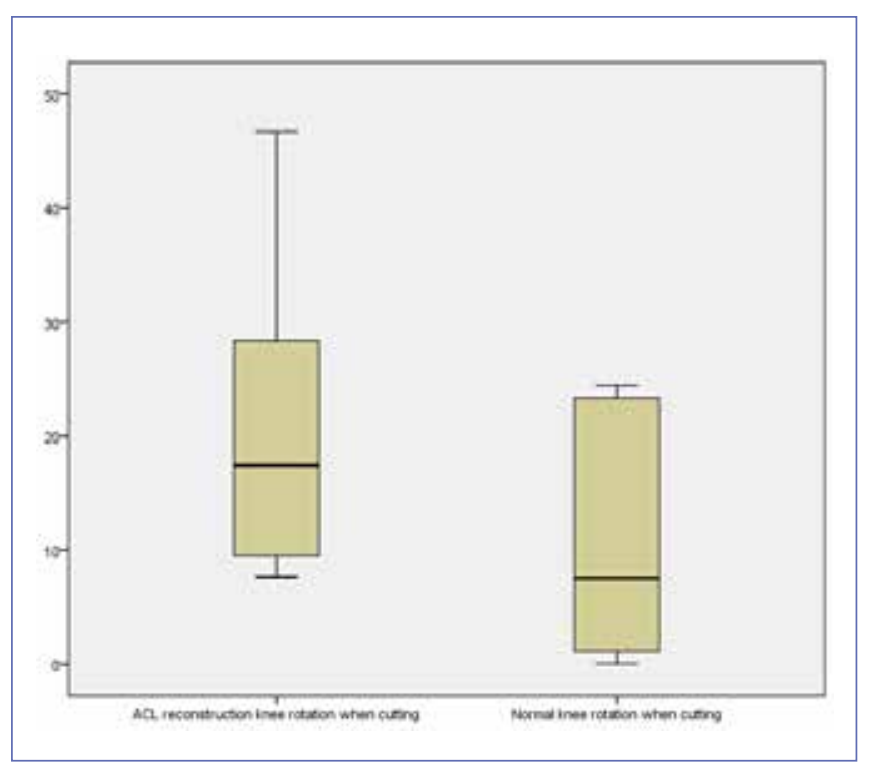

Figure 10 - Knee rotation while performing the cutting test. The vertical line represents the knee rotation in degrees.

Table III - Knee rotation while performing the weaving test.

\begin{tabular}{lll}
\hline & $\begin{array}{l}\text { ACL reconstructed } \\
\text { knee }\end{array}$ & $\begin{array}{l}\text { Non-operated } \\
\text { knee }\end{array}$ \\
\hline $\begin{array}{l}\text { mean rotation }(\mathbf{d} \theta / \\
\mathbf{d T i m e})\end{array}$ & $17.88 \pm 9.83$ & $21.34 \pm 11.14$ \\
\hline $\mathbf{t}$ & 0.929 & \\
\hline $\mathbf{d f}$ & 4 & \\
\hline $\mathbf{p}(\mathbf{2}$ tailed $)$ & 0.406 & \\
\hline
\end{tabular}

Values for rotation are in degrees. No significant differences were found. 


\section{DISCUSSION}

This study evaluated whether ACL reconstructed knees exhibit gait disturbances and whether these gait disturbances occurred in cutting and weaving movements. The participants answered the Lysholm scoring questionnaire at the beginning of the study to evaluate the outcome of the knee ligament surgery, the results being fair to good.

When comparing the knee which had undergone an ACL reconstruction surgery with the contralateral, there seemed to be greater variation in the movement of the ACL reconstructed knee, which showed a greater internal rotation while walking when compared to the non-operated knee, where the movement was more controlled. There could be more joint laxity in the ACL reconstructed knee compared to the non-operated knee loss of sensory information, which could result in errors in the joint coordinative patterns during gait (11). Other studies observed that healthy controls and ACL reconstructed individuals exhibited different knee joint coordination while walking (9). They also noticed that there were some additional differences between the shank-thigh phase dynamics while walking which were most pronounced in the late portion of the stance period, where ACL reconstructed subjects had a more out of phase relationship (9). Primary ACL injury occurs during early deceleration in cutting and landing movements (6). The cutting task showed that the rotation in the knee of the ACL reconstructed participants is larger than in the non-operated knees. The sample size was smaller than the minimum of ten subjects per group to have a statistical power of $80 \%$. Stearns et al. found that female soccer players have abnormal frontal plane knee mechanics during sidestep cutting (6). Their female soccer players increased abduction angles and knee adductor moments post ACL reconstruction surgery (6). A study regarding postural adjustments following ACL rupture and reconstruction showed that compensatory postural responses of the lower limb to unpredictable perturbations of the knee joint were altered/delayed in ACL reconstructed participants when compared to healthy participants, while anticipatory responses to predictable perturbations were not different between ACL reconstructed and healthy participants. The study suggested that the earliest goals after ACL reconstruction should be to target compensatory adjustments in order to improve functional stabilisation of the knee joint by the time of return to sport (12).
During the weaving task, we found no difference in the late stance knee rotation between the ACL reconstructed knees and the non-operated knees. However, we caution that the sample size might have been too small.

There was better reliability on the right knees than on the left knees. This might have resulted from a visibility issue of the markers on the left knees. Reliability will need to be more robust to be able to obtain more "clean" data. The control group will need to be retested. A retest of the control group might present a better intra-class correlation coefficient. If the correlation coefficients will not appear to be improved, a method to handle improved (normalized) indicators which would better define the classes should be tested.

Another major limitation was the small sample size. Further studies would need to increase the number of participants. Also, the laboratory environment cannot recreate the aggressive movements employed during sports activity this study tested subjects while walking. Further research should include movements which are closer to those performed on sports fields and should also investigate the delay between compensatory postural responses and the neuromechanical deficits observed during demanding functional and sports specific movements such as running and landing tasks.

\section{CONCLUSION}

There were no evident gait disturbances between reconstructed knees and healthy contralateral knees in sportsmen who have undergone ACL reconstruction surgery. Furthermore, although there is an increased rotation of the knee during walking and cutting activity in ACL reconstructed knees, statistical analyses revealed no significance. We however point out that these results should be regarded as preliminary, given, for example, the mall sample size which was recruited.

For further studies, a larger sample size would provide more conclusive and significant results to clarify whether there are differences between the ACL reconstructed and the non-operated knees. Reliability will need to be more robust to be able to obtain more definitive data. The control group will need to be retested.

\section{Funding}

The authors declare that they have no conflict of interests, financial or otherwise, with regard to this paper. 


\section{REFERENCES}

1. Lohmander LS, Ostenberg A, Englund M, Roos H. High prevalence of knee osteoarthritis, pain, and functional limitations in female soccer players twelve years after anterior cruciate ligament injury. Arthritis Rheum 2004; 50:3145-3152.

2. Delahunt E, Prendiville A, Sweeney L, Chawke M, Kelleher J, Patterson M, Murphy K. Hip and knee joint kinematics during a diagonal jump landing in anterior cruciate ligament reconstructed females. J Electromyogr Kinesiol 2012; 22(4):598-606.

3. Jomha NM, Pinczewski LA, Clingeleffer A et al. Arthroscopic reconstruction of the anterior cruciate ligament with patellar-tendon autograph and interference screw fixation. The results of seven years. J Bone Joint Surg 1999; 81B:775-779.

4. Roewer BD, Di Stasi SL, Snyder-Mackler L. Quadriceps strength and weight acceptance strategies continue to improve two years after anterior cruciate ligament reconstruction. J Biomech 2011; 44:1948-1953.

5. Paterno MV, Schmitt LC, Ford KR, et al. Biomechanical measures during landing and postural stability predict second anterior cruciate ligament injury after anterior cruciate ligament reconstruction and return to sport. Am J Sports Med 2010; 38:1968-1978.

6. Stearns KM, Pollard CD. Abnormal frontal plane knee mechanics during sidestep cutting in female soccer athletes after anterior cruciate ligament reconstruction and return to sport. Am J Sports Med 2013; 41(4):918-923.

7. Vairo GL, Myers JB, Sell TC, Fu FH, Harner CD, Lephart SM. Neuromuscular and biomechanical landing performance subsequent to ipsilateral semitendinosus and gracilis autograft anterior cruciate ligament reconstruction. Knee Surg Sports Traumatol Arthrosc 2008; 16(1):2-14.

8. Butler RJ, Minick KI, Ferber R, Underwood F. Gait mechanics after ACL reconstruction: implications for the early onset of knee osteoarthritis. Br J Sports Med 2009; 43:366-370.

9. Kurz MJ, Stergiou S, Buzzi UH, Georgoulis AD. The effect of anterior cruciate ligament reconstruction on lower extremity relative phase dynamics during walking and running; Knee Surg Sports Traumatol Arthrosc 2005; 13:107-115.

10. Padulo J, Oliva F, Frizziero A, Maffulli N. Muscles, Ligaments and Tendons Journal - Basic principles and recommendations in clinical and field Science Research: 2016 Update. MLTJ 2016; 6(1):1-5.

11. Johansson H, Sjolander P, Sojka P.A sensory role for cruciate ligaments. Clin Orthop Rel Res 1991; 268:161-178.

12. Labanca L, Laudani L, PaoloPM, Macaluso A. Postural Adjustments Following ACL Rupture and Reconstruction: A Longitudinal Study. Int J Sports Med 2018; 39: 549-554.

13. Papalia R, Franceschi F, Tecame A, D’Adamio S, Maffulli N, Denaro V. Anterior cruciate ligament reconstruction and return to sport activity: postural control as the key to success. Int Orthop 2015; 39(3):527-534.

14. Longo UG, Rizzello G, Frnaceschi F, Campi S, Maffulli N, Denaro V. The architecture of the ipsilateral quadriceps two years after successful anterior cruciate ligament reconstruction with bone-patellar tendon-bone autograft. Knee 2014; 21(3):721-725.

15. Di Vico G, Di Donato SL, Balato G, Correra G, D’Addona A, Maffulli N, Rosa D. Correlation between time from injury to surgery and the prevalence of ramp and hidden lesions during anterior cruciate ligament reconstruction. A new diagnostic algorithm. MLTJ 2018; 7(3):491-497.

16. Maffulli N, Osti L. ACL stability, function, and arthritis: what have we been missing? Orthopedics 2013; 36(2):90-92.

17. Maffulli N, Loppini M, King JB. Anterior cruciate ligament tears: what we already know. Knee Surg Sports Traumatol Arthrosc 2013; 21(7):1704-1705.

18. Osti L, Papalia R, Del Buono A, Amato C, Denaro V, Maffulli N. Good results five years after surgical management of anterior cruciate ligament tears, and meniscal and cartilage injuries. Knee Surg Sports Traumatol Arthrosc 2010; 18(10):1385-1390.

19. Volpi P, Bisciotti GN, Chamari K, Cena E, Carimati G, Bragazzi NL. Risk factors of anterior cruciate ligament injury in football players: a systematic review of the literature. MLTJ 2016; 6(4):480-485.

20. Oberhofer K, Hosseini Nasab SH, Schütz P, Postolka B, Snedeker JG, Taylor WR, List R. The influence of muscle-tendon forces on ACL loading during jump landing: a systematic review. MLTJ 2017; 7(1):125-135.

21. Balachandar V, Marciniak JL, Wall O, Balachandar C. Effects of the menstrual cycle on lower-limb biomechanics, neuromuscular control, and anterior cruciate ligament injury risk: a systematic review. MLTJ 2017; 7(1):136-146.

22. Karagiannidis E, Kellis E, Galanis N, Vasilios B.Semitendinosus muscle architecture during maximum isometric contractions in individuals with anterior cruciate ligament reconstruction and controls. MLTJ 2017; 7(1):147-151.

23. Trinchese GF,Oliva F, Maffulli N. Minimally invasive anatomic reconstruction of the anterolateral ligament with ipsilateral gracilis tendon. MLTJ 2017; 7(2):240-246.

24. Tudisco C, Bisicchia S, Cosentino A, Chiozzi F, Piva M. Knee stability, athletic performance and sport-specific tasks in non-professional soccer players after ACL reconstruction: comparing trans-tibial and antero-medial portal techniques. MLTJ 2015; 5(3):175-180.

25. Dhillon MS, Bali K, Prabhakar S. Differences among mechanoreceptors in healthy and injured anterior cruciate ligaments and their clinical importance. MLTJ. 2012; 2(1):38-43.

26. Vasta S, Papalia R, Albo E, Maffulli N, Denaro V. Top orthopaedic sports medicine procedures. J Orthop Surg Res 2018; 13(1):190.

27. Papandreou MG, Billis EV, Antonogiannakis EM, Papaioannou NA. Effect of cross exercise on quadriceps acceleration reaction time and subjective scores (Lysholm questionnaire) following anterior cruciate ligament reconstruction. J Orthop Surg Res 2009 J; 4:2. 